\title{
A precessing accretion disc in the intermediate polar $\mathrm{XY}$ Arietis?
}

\author{
A. J. Norton ${ }^{1}$ and K. Mukai ${ }^{2}$ \\ 1 Department of Physics and Astronomy, The Open University, Walton Hall, Milton Keynes MK7 6AA, UK \\ e-mail: A. J . Norton@open.ac.uk \\ 2 CRESST and X-ray Astrophysics Laboratory NASA/GSFC, Greenbelt, MD 20771, USA and Department of Physics, University of \\ Maryland, Baltimore county, 1000 Hilltop Circle, Baltimore, MD 21250, USA \\ e-mail: mukai@milkyway.gsfc.nasa.gov
}

Received 30 April 2007 / Accepted 23 June 2007

\section{ABSTRACT}

\begin{abstract}
Context. XY Ari is the only intermediate polar to show deep X-ray eclipses of its white dwarf. Previously published observations with Ginga and Chandra have also revealed a broad X-ray orbital modulation, roughly antiphased with the eclipse, and presumed to be due to absorption in an extended structure near the edge of an accretion disc. The X-ray pulse profile is generally seen to be double-peaked, although a single-peaked pulse was seen by RXTE during an outburst in 1996.

Aims. We intended to investigate the cause of the broad orbital modulation in XY Ari to better understand the accretion flow in this system and other intermediate polars.

Methods. We observed XY Ari with RXTE and analysed previously unpublished archival observations of the system made with ASCA and XMM-Newton. These observations comprise six separate visits and span about ten years.

Results. The various X-ray observations show that the broad orbital modulation varies in phase and significance, then ultimately disappears entirely in the last few years. In addition, the X-ray pulse profile shows variations in depth and shape, and in the recent $R X T E$ observations displays no evidence for changes in hardness ratio.

Conclusions. The observed changes indicates that both the pulse profile and the orbital modulation are solely due to geometrical effects at the time of the RXTE observations, rather than phase-dependent variations in photoelectric absorption as seen previously. We suggest that this is evidence for a precessing, tilted accretion disc in this system. The precession of the disc moves structures out of our line of sight both at its outer edge (changing the orbital modulation) and at its inner edge where the accretion curtains are anchored (changing the pulse profile).
\end{abstract}

Key words. stars: novae, cataclysmic variables - X-rays: binaries - stars: magnetic fields - stars: binaries: eclipsing stars: individual: XY Ari - accretion, accretion discs

\section{Introduction}

XY Ari is an intermediate polar (IP), namely a semi-detached interacting asynchronous binary star in which a magnetic white dwarf with a surface field strength of order a few megagauss, accretes material from a Roche lobe-filling, usually main sequence dwarf, companion. The accretion flow from the donor star generally forms a truncated accretion disc whose inner edge is set by the white dwarf's magnetospheric radius. Here the material attaches to the magnetic field lines before following them towards the white dwarf magnetic poles. Arc-shaped accretion curtains are believed to form, standing above the white dwarf surface. Towards the base of these curtains, the accretion flow undergoes a strong shock, below which material settles onto the white dwarf, releasing X-ray to optical emission. Since the magnetic axis is offset from the spin axis of the white dwarf, this gives rise to the defining characteristic of the class, namely $\mathrm{X}$-ray (and usually optical) emission pulsed at the white dwarf spin period. Around 30 confirmed IPs are now recognised, with a further 20 or so candidate systems having been proposed ${ }^{1}$. Comprehensive reviews of various aspects of their behaviour are given by Patterson (1994), Warner (1995) and Norton et al. (2004).

The pulse profiles of IPs are variously seen to be singlepeaked (e.g. AO Psc, V1223 Sgr, TX Col, EX Hya, etc.),

\footnotetext{
1 See http://asd.gsfc.nasa.gov/Koji.Mukai/iphome/ iphome.html for an on-line catalogue of systems.
}

double-peaked (YY Dra, V405 Aur, GK Per, etc.), or displaying more complex shapes (FO Aqr, BG CMi, PQ Gem, etc.). It is apparent that most of these profile shapes are due to a combination of emission from both upper and lower accreting magnetic poles of the white dwarf. Depending on the geometry and the relative column density parallel to and perpendicular to the white dwarf surface, either a single-peaked or double-peaked profile can result (Norton et al. 1999). Introducing asymmetries between the two poles can yield more complex profiles (Beardmore et al. 1998). During outbursts, GK Per and XY Ari have each been seen to develop a single-peaked pulse profile (Watson et al. 1985; Hellier et al. 1997). This is interpreted as arising when the lower pole becomes hidden by the inner extension of the outbursting accretion disc as it forces the magnetosphere inwards (Hellier et al. 1997; Hellier et al. 2004).

Around half of the known IPs display orbitally modulated X-ray flux (Parker et al. 2005). The cause of this modulation is generally believed to be local absorption of X-rays in an extended structure at the outer edge of the accretion disc, caused by the impact of the stream from the inner Lagrangian point with the disc itself. Absorption at the outer edge of a disc is the accepted explanation for X-ray orbital modulations seen in Low Mass X-ray Binaries too. In our systematic study of orbital modulation in sixteen IPs, covering thirty observations with $R X T E$ and $A S C A$, we noted that the presence of orbital modulation can appear and disappear on timescales of years or months in an individual system. This was particularly apparent in AO Psc and 
V1223 Sgr, possibly because these have been some of the best studied systems over the years so there is a wealth of observational data sets for each system, capturing its behaviour at many different epochs.

\section{The history of $X Y$ Ari}

$\mathrm{XY}$ Ari was originally discovered as the X-ray source $1 \mathrm{H} 0253+193$ by the Einstein satellite (Halpern \& Patterson 1987). Subsequent short Ginga observations in July 1987 and January 1989 discovered a coherent X-ray pulsation with a period of $206 \mathrm{~s}$ and a double-peaked profile (Takano et al. 1989; Koyama et al. 1991), suggesting its identification as an IP (Patterson \& Halpern 1990). Further, longer observations with the same satellite in August 1989 discovered eclipses with a period of $6.06 \mathrm{~h}$ (Kamata et al. 1991), so establishing the orbital period of the system and making it the first (and so far only) IP to exhibit deep X-ray eclipses.

Since XY Ari lies behind the molecular cloud MBM12, no optical counterpart can be seen, but the system is detected in the infrared, where an ellipsoidal modulation is apparent (Allan et al. 1996). Modelling of this lightcurve allows determination of the system parameters. In particular the mass ratio is determined to lie in the range $0.43<q<0.71$ and the system inclination angle in the range $80^{\circ}<i<87^{\circ}$. Infrared spectroscopy of XY Ari has allowed its distance to be estimated as $270 \pm 100 \mathrm{pc}$ (Littlefair et al. 2001).

The eclipsing nature of XY Ari is important, as accurate timing of the eclipse ingress and egress allows a determination of the size of the X-ray emitting region on the WD surface. Hellier (1997) used a series of short RXTE observations, spanning many eclipse egresses, to show that the emitting region has a size smaller than 0.002 of the WD surface area, and that the accretion region itself may wander over a larger area, of fractional size less than 0.01 of the WD surface, on a timescale comparable to the orbital period. Hellier (1997) also used this study of the eclipse to constrain the upper limit on the inclination angle further, namely $i<84^{\circ}$.

A further interesting aspect of the behaviour of XY Ari was revealed by these same short RXTE observations, namely that the source exhibitted an outburst in July 1996, during which the mass accretion rate increased substantially (Hellier et al. 1997). The outburst was consistent with being due to a thermal-viscous disc instability, as in conventional dwarf novae, although alternatives, such as an enhanced mass transfer rate from the secondary, could not be ruled out.

Also of interest for the present study is the fact that the system displays a broad, sinusoidal X-ray orbital modulation, on which the deep X-ray eclipse is superimposed. In the Ginga observations from 1989, Kamata et al. (1991) show that the minimum of the broad modulation lags the narrow eclipse by about 0.45 in orbital phase, with the eclipse occurring just after the maximum of the broad modulation. They further demonstrate that the broad modulation is antiphased with the X-ray hardness ratio, implying that the cause of the modulation is photoelectric absorption. The only other published X-ray observations of XY Ari of sufficient duration to observe the full orbital cycle are those by Chandra in July/August 2000, reported by Salinas $\&$ Schlegel (2004). Although only covering parts of four orbital cycles, and of lower signal-to-noise than the Ginga observations, these lightcurves too show the broad orbital modulation. The phasing here is such that the broad orbital minimum lags the deep eclipse by $\sim 0.65-0.75$ in orbital phase, with the eclipse occurring just before the maximum of the broad X-ray modulation. Based on just these two observations therefore, there is already evidence for variability in the broad X-ray modulation component in this system.

\section{Observations and data reduction}

\subsection{New RXTE observations}

The new data presented here were obtained with the PCA instrument on the RXTE satellite. The observation sequence comprises eight segments, each of about 6 hours duration on the 19th, 20th, 23rd and 24th November 2005 and on the 7th, 8th, 10th and 11th January 2006. Each of the eight segments comprises data from four satellite orbits, resulting in typically $\sim 11 \mathrm{ks}$ of usable on-source data from each. Various combinations of the 5 proportional counter units of the PCA were active during each observation segment. Neglecting PCU0, for which the background subtraction is problematical since the loss of the propane layer, data from either 1, 2 or 3 PCUs were available at any time.

Data were extracted using standard criteria from the top layer only of each PCU, to make lightcurves with $16 \mathrm{~s}$ time resolution, in each of four energy ranges, namely $2-4 \mathrm{keV}, 4-6 \mathrm{keV}$, 6-10 keV and 10-20 keV. Background lightcurves were generated using the latest RXTE background models and subtracted from the on source lightcurves. To account for the different number of PCUs active at any time, the resulting count rates were simply scaled by the number of active PCUs, to yield counts per second per PCU.

The power spectra of the resulting lightcurves, both from the separate November and January visits and from the combined observation, are dominated by signals at high harmonics of the orbital frequency, due to the narrow X-ray eclipse. There is no signal at the fundamental orbital frequency in the power spectra of any of the lightcurves. Signals at the spin frequency of the white dwarf, and its first harmonic, are only marginally significant.

The lightcurves for the two halves of the observation (from November 2005 and January 2006), folded at the 206 s white dwarf spin period are shown in Fig. 1. Although four energy ranges were extracted, as noted above, we show here the combined 2-10 keV lightcurve for ease of comparison with other datasets presented in later sections. The familiar double peaked pulse profile is seen in each energy band, albeit with a rather poor signal-to-noise ratio. Phase zero is arbitrary in these profiles. We fitted a sinusoid to these data with a period equal to half the white dwarf spin period, but with the amplitude, mean level and phase of minimum flux as free parameters in each case. This yielded the curves which are over-plotted in Fig. 1. The depths of this modulation are listed in Table 1 and defined as the peak-to-peak range of the sinusoid divided by the maximum value. The modulation depths here are all rather low, at around $10 \%-15 \%$ at all energies at both epochs. The constancy of the modulation depth is further demonstrated by the fact that the $(2-6 \mathrm{keV} / 6-10 \mathrm{keV})$ hardness ratio is constant when folded at the spin period.

The RXTE lightcurves folded at the $6.06 \mathrm{~h}$ orbital period are shown in Fig. 2. As for the pulse profiles, although lightcurves were extracted in four separate energy bands, we show here the combined 2-10 keV orbital profile for ease of comparison with other datasets presented in later sections. The narrow eclipse is clearly present, with the X-ray flux virtually extinguished for $\sim 0.15$ of the orbital cycle, but the broad orbital modulation seen in previous observations has essentially disappeared. As for the pulse profiles, we fitted a sinusoid to these data (excluding the 

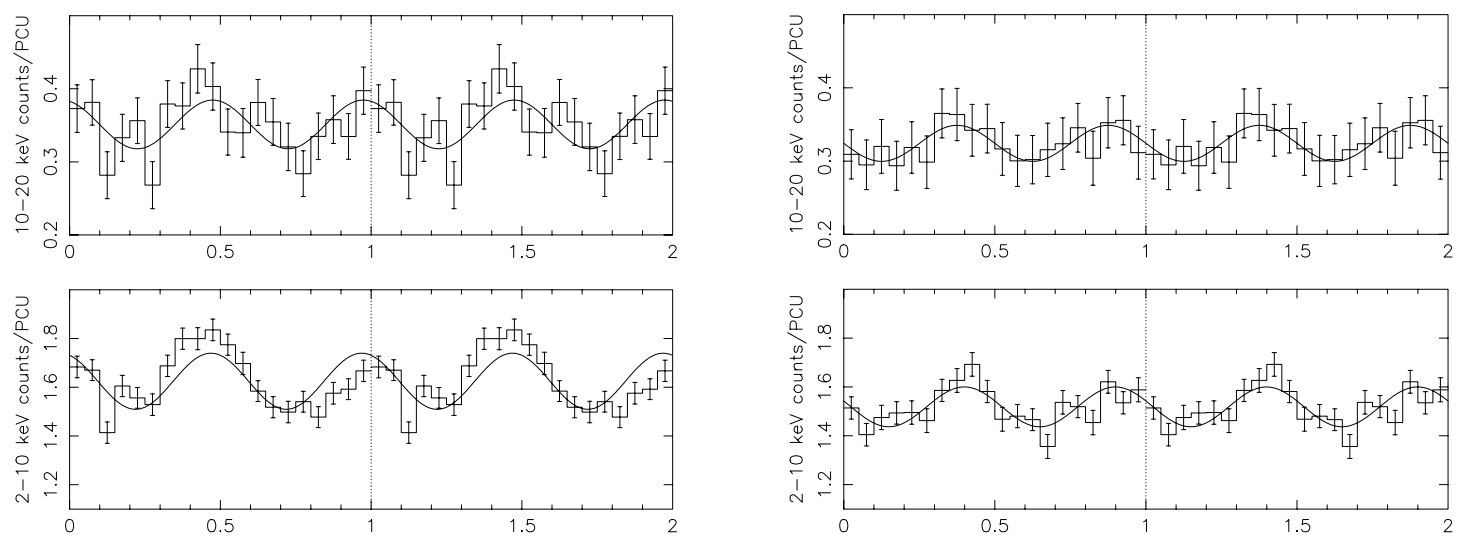

Fig. 1. The RXTE X-ray lightcurves of XY Ari from November 2005 (left) and January 2006 (right) folded at the $206 \mathrm{~s}$ white dwarf spin period. In the upper panel the energy range is $10-20 \mathrm{keV}$, whilst in the lower panel it is $2-10 \mathrm{keV}$. The sinusoids overplotted are the best fit to any double peaked pulse profile.
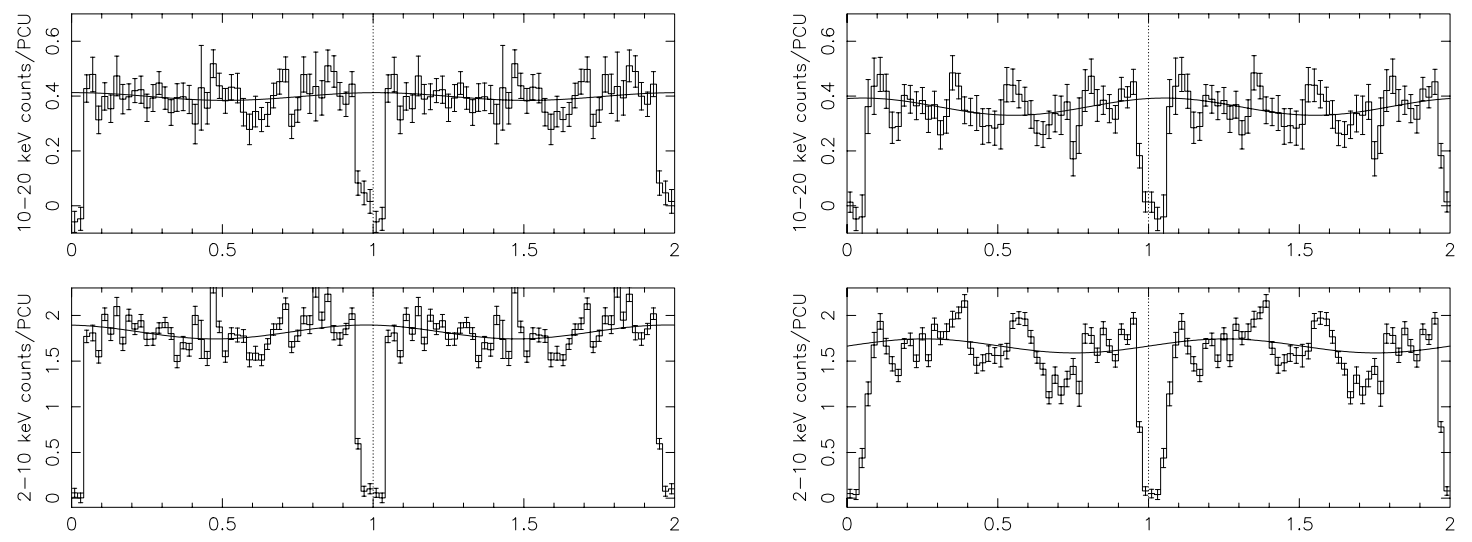

Fig. 2. The RXTE X-ray lightcurves of XY Ari from November 2005 (left) and January 2006 (right) folded at the $6.06 \mathrm{~h}$ orbital period of the binary. In the upper panel the energy range is $10-20 \mathrm{keV}$, whilst in the lower panel it is $2-10 \mathrm{keV}$. The sinusoids overplotted are the best fit to any broad modulation, excluding the eclipse itself.

Table 1. The double-peaked modulation depth of the pulse profile in various X-ray observations of XY Ari.

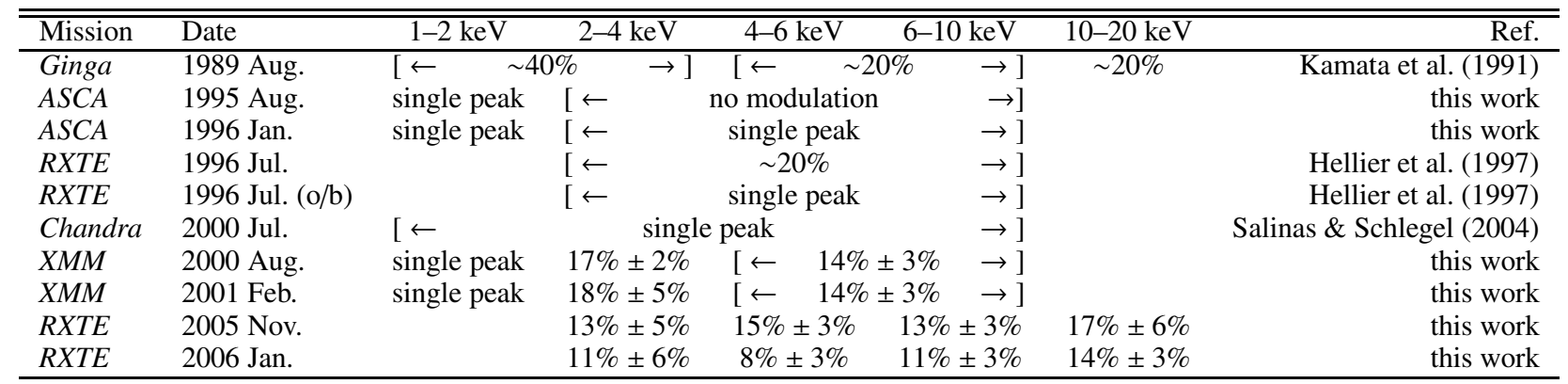

eclipse itself), with the amplitude, mean level and phase of minimum flux as free parameters in each case. This yielded the curves which are over-plotted in Fig. 2; the depths of the broad modulation are listed in Table 2. For these RXTE observations, the modulation depth in all energy bands is consistent with zero at the $\sim 2 \sigma$ level.

Fitting a simple model to the average spectra of the November 2005 and January 2006 visits reveals that both are adequately fitted (with $\chi_{r}^{2}=0.77$ in each case) by a thermal bremsstrahlung model with an iron line. The temperature is $k T=$ $35_{-9}^{+15} \mathrm{keV}$ in the first observation and $k T=38_{-10}^{+20} \mathrm{keV}$ in the second, with hydrogen column densities of $N_{\mathrm{H}}=5.3_{-1.2}^{+1.1} \times 10^{22} \mathrm{~cm}^{-2}$ and $N_{\mathrm{H}}=6.7_{-1.3}^{+1.4} \times 10^{22} \mathrm{~cm}^{-2}$ respectively. The spectra are therefore consistent with each other. The $2-10 \mathrm{keV}$ flux however, shows a slight reduction from $1.45 \times 10^{-11} \mathrm{erg} \mathrm{cm}^{2} \mathrm{~s}^{-1}$ to $1.32 \times 10^{-11} \mathrm{erg} \mathrm{cm}^{2} \mathrm{~s}^{-1}$, but there are likely to be systematic uncertainties of up to $5 \%$ on these values due to background subtraction, judging by the deviations from zero flux at mid eclipse.

\subsection{Archival ASCA observations}

There are two archival ASCA observations of XY Ari, neither of which have previously been published, except in compilation papers examining IP X-ray spectra (Ezuka \& Ishida 1999; Terada et al. 2004). They comprise an observation from 1995 August 6 with an exposure of around $35 \mathrm{ks}$ and another from 

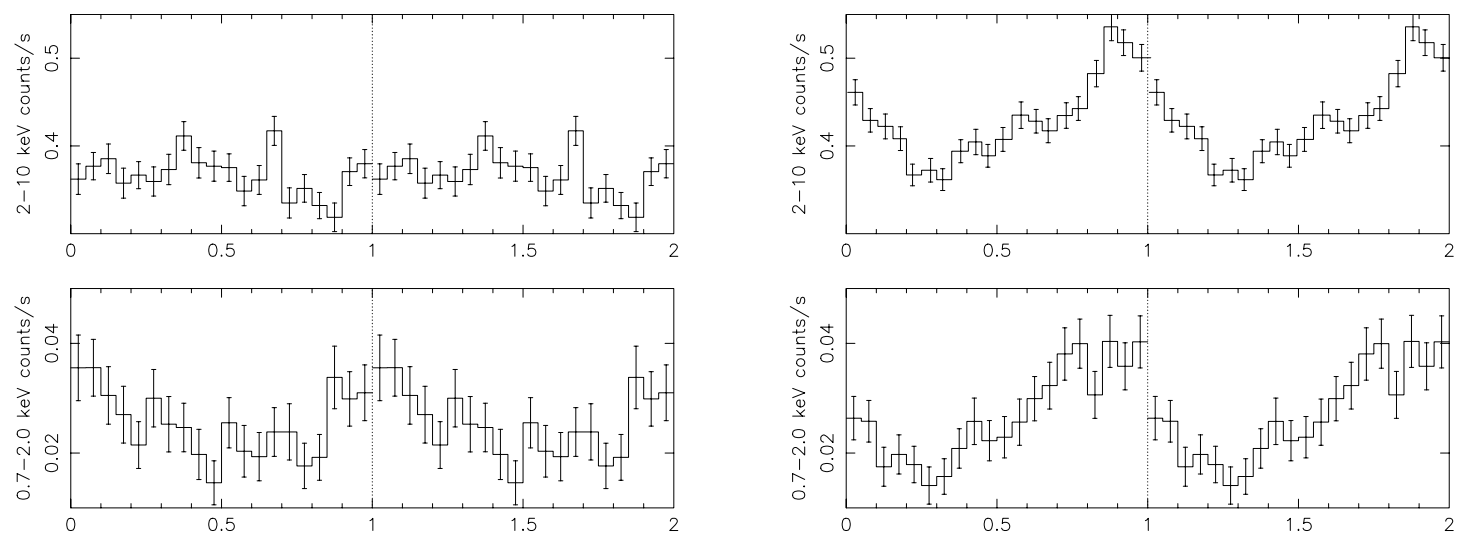

Fig. 3. The ASCA X-ray lightcurves of XY Ari from August 1995 (left) and January 1996 (right) folded at the $206 \mathrm{~s}$ white dwarf spin period. The energy range of the upper lightcurve is $2-10 \mathrm{keV}$, whilst that of the lower lightcurve is $0.7-2.0 \mathrm{keV}$.
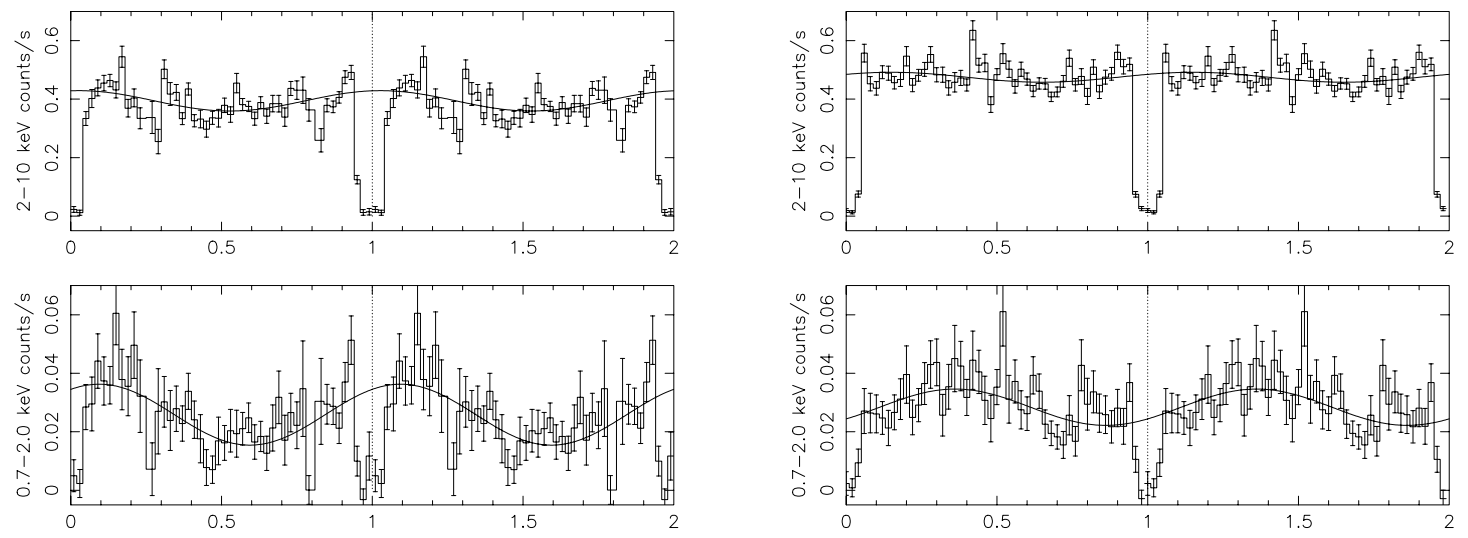

Fig. 4. The ASCA X-ray lightcurves of XY Ari from August 1995 (left) and January 1996 (right) folded at the $6.06 \mathrm{~h}$ orbital period of the binary. The energy range of the upper lightcurve is $2-10 \mathrm{keV}$, whilst that of the lower lightcurve is $0.7-2.0 \mathrm{keV}$. The sinusoid overplotted is the best fit to any broad modulation, excluding the eclipse itself.

Table 2. The depth and phase of minimum of the broad orbital modulation in various X-ray observations of XY Ari.

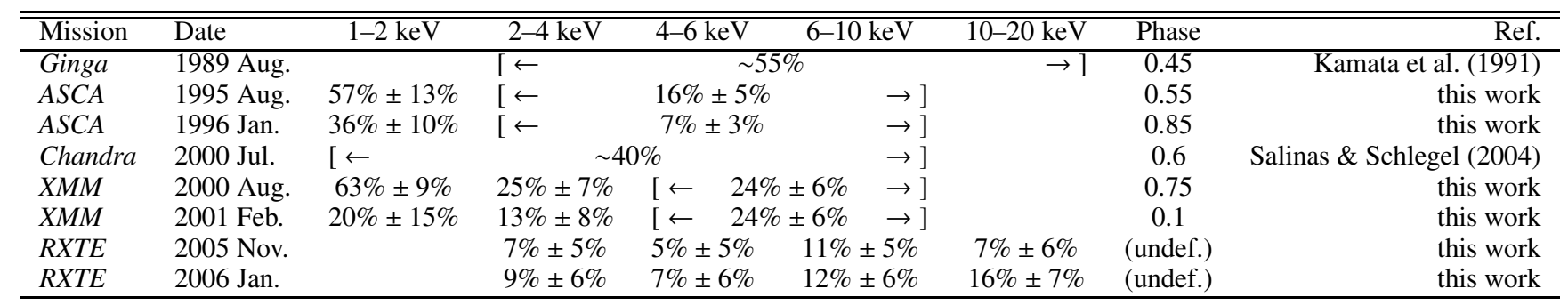

1996 January 28 with an exposure of around $60 \mathrm{ks}$. We extracted lightcurves in each case from both the SIS and GIS detectors, in two energy bands. The low energy band corresponds to $\sim 0.7-2.0 \mathrm{keV}$ whilst the high band is roughly $\sim 2-10 \mathrm{keV}$.

Background subtracted lightcurves obtained by summing the lightcurves from the two SIS and two GIS instruments, folded at the white dwarf spin period in each case are shown in Fig. 3. As in Fig. 1, phase zero is arbitrary in these plots. In the August 1995 data there is little evidence for any modulation above $2 \mathrm{keV}$, although a possible single-peaked pulse is visible at low energies. By contrast, in January 1996, there is a clear single-peaked pulse profile in both energy bands.

The lightcurves folded at the orbital period are shown in Fig. 4. As in the case of Fig. 2, we show the best fit sinusoid to these folded lightcurves (excluding the eclipse) overplotted. Here the depths of the modulation, listed in Table 2, are significant at the $\sim 4 \sigma$ level in the low energy band, with a more marginal detection in the high energy band.

The spectra of these ASCA data are also adequately fit by simple thermal bremsstrahlung models, with $\chi_{r}^{2}=0.70$ and 0.78 respectively. The $2-10 \mathrm{keV}$ fluxes are consistent across the two observations at $1.79 \times 10^{-11} \mathrm{erg} \mathrm{cm}^{2} \mathrm{~s}^{-1}$ and $1.84 \times$ $10^{-11} \mathrm{erg} \mathrm{cm}^{2} \mathrm{~s}^{-1}$, with likely statistical uncertainties of order $\sim 1 \%$. The fitted column densities too are consistent with each other with values of $N_{\mathrm{H}}=4.1_{-0.2}^{+0.3} \times 10^{22} \mathrm{~cm}^{-2}$ and $N_{\mathrm{H}}=$ $4.4 \pm 0.1 \times 10^{22} \mathrm{~cm}^{-2}$ respectively.

\subsection{Archival XMM-Newton observations}

There are also two archival XMM-Newton observations of XY Ari, neither of which have previously been published either. They comprise an observation from 2000 August 26 with 

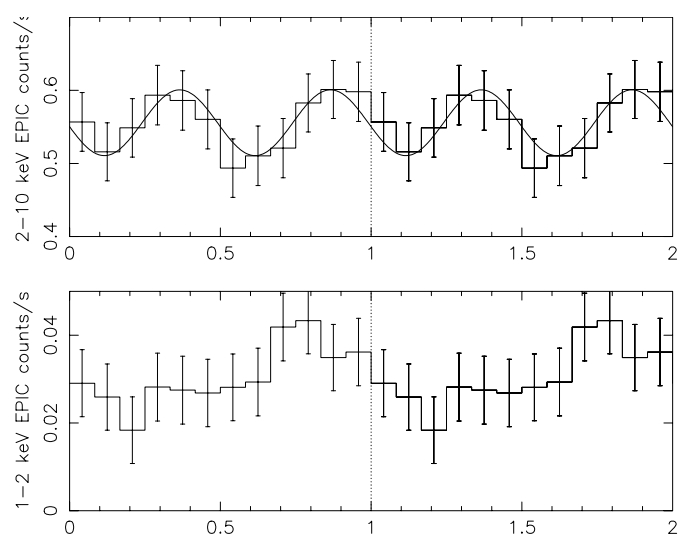
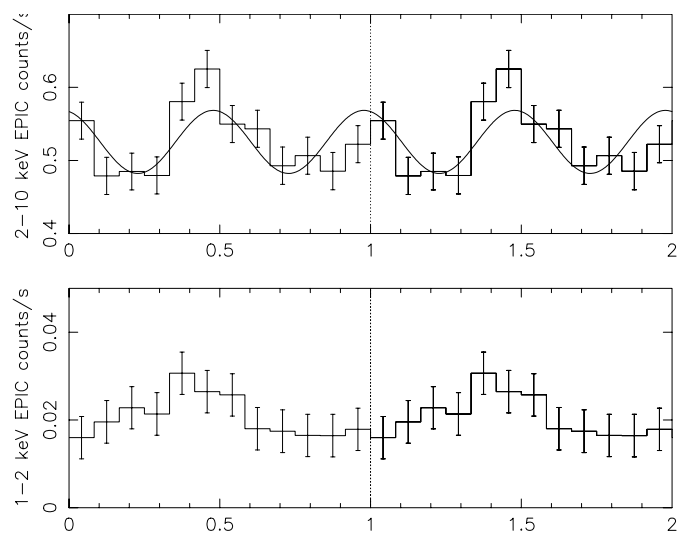

Fig. 5. The XMM-Newton combined PN and MOS X-ray lightcurves of XY Ari from August 2000 (left) and February 2001 (right) folded at the $206 \mathrm{~s}$ white dwarf spin period. Count rates are scaled to that of the PN detector only in each case. The energy range of the upper lightcurve is $2-10 \mathrm{keV}$, whilst that of the lower lightcurve is $1-2 \mathrm{keV}$. The sinusoids overplotted are the best fit to any double peaked pulse profile.
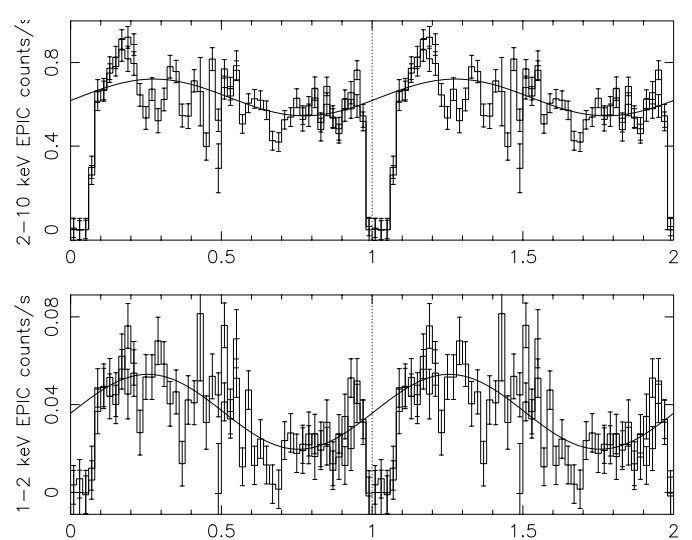
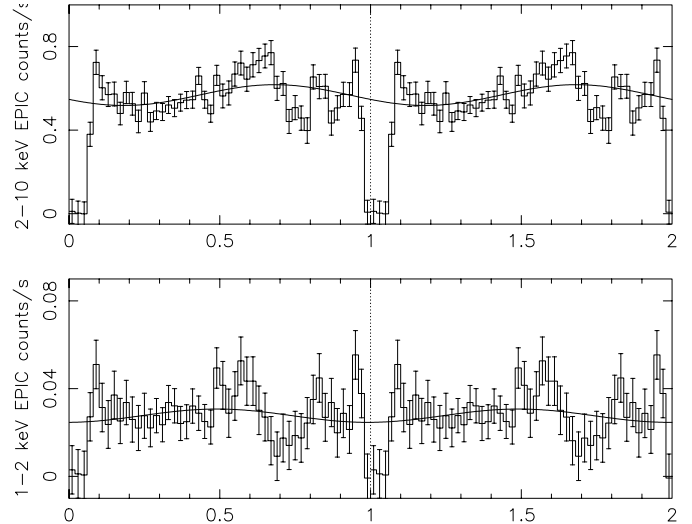

Fig. 6. The XMM-Newton combined PN and MOS X-ray lightcurves of XY Ari from August 2000 (left) and February 2001 (right) folded at the $6.06 \mathrm{~h}$ orbital period of the binary. The energy range of the upper lightcurve is $2-10 \mathrm{keV}$, whilst that of the lower lightcurve is $1-2 \mathrm{keV}$. The sinusoid overplotted is the best fit to any broad modulation, excluding the eclipse itself. In the 2000 data, there is little phase overlap between the MOS and PN data, so these are plotted separately in this case. The 2001 data are scaled to the count rate of the PN only to facilitate comparison.

exposures of around $16 \mathrm{ks}(\mathrm{MOS})$ and $18 \mathrm{ks}(\mathrm{PN})$ and another from 2001 February 5 with exposures of around 29 ks (MOS) and $27 \mathrm{ks}(\mathrm{PN})$. We extracted lightcurves in each case from both the MOS and PN detectors, in three energy bands. The low energy band corresponds to $\sim 1-2 \mathrm{keV}$, the medium energy band is $\sim 2-4 \mathrm{keV}$, whilst the high band is $\sim 4-10 \mathrm{keV}$. In the observation from 2001 February, XY Ari fell on a chip gap on the MOS1 detector, so the lightcurves are extracted from the PN and MOS2 only.

Background subtracted lightcurves combined from the MOS and PN instruments and folded with an arbitrary phase zero at the white dwarf spin period, are shown in Fig. 5. To allow comparison between the two epochs, these lightcurves are each scaled to the count rate from the PN detector only. As for the $R X T E$ data, we show the combined $2-10 \mathrm{keV}$ data to facilitate comparison with the other observations presented here. In these pulse profiles the expected double-peaked shapes are seen above $2 \mathrm{keV}$ in each observation, but the low energy pulse profile is apparently single-peaked at each epoch. As in Fig. 1, the best-fitting sinusoid with a period equal to half the white dwarf spin period is over-plotted on the higher energy lightcurves. The modulation depths, listed in Table 1, are consistent between the two observations and between the two higher energy bands.

Figure 6 shows the same lightcurves, folded at the binary orbital period. In the case of the 2000 observation, there is very little orbital phase overlap between the MOS and PN lightcurves, so we show these as separate points. In the 2001 observation there is good orbital phase overlap between the MOS and PN lightcurves, so in Fig. 6 these are averaged together. As with Fig. 5, these lightcurves are each scaled to the count rate of the PN detector only, to allow comparison between the two epochs, and the two higher energy bands extracted are combined into a single 2-10 keV lightcurve. As with Figs. 2 and 4, we show the best fit sinusoid to these folded lightcurves (excluding the eclipse) overplotted. Here the depths of the modulation, listed in Table 2, are significant at the $\sim 4 \sigma$ level or greater in each energy band during the 2000 observation, but during the 2001 observation the modulation is not so clearly detected. These $X M M-N e w t o n$ folded lightcurves show much more variation at all phases than the RXTE data, with hints of a structured modulation as a function of orbital phase.

As with the other observations presented here, the spectra of these $X M M$ data are adequately fit by simple thermal bremsstrahlung models, with $\chi_{r}^{2}=1.07$ and 1.11 respectively. The $2-10 \mathrm{keV}$ fluxes are similar for the two observations at $1.13 \times 10^{-11} \mathrm{erg} \mathrm{cm}^{2} \mathrm{~s}^{-1}$ and $1.20 \times 10^{-11} \mathrm{erg} \mathrm{cm}^{2} \mathrm{~s}^{-1}$, with likely statistical uncertainties of order $\sim 1 \%$. We note, however, that there is incomplete orbital phase coverage during the observation in 2000 August, so the flux is likely to be an under-estimate of the true mean value, as the phase range sampled includes the eclipse, but not all of the out-of-eclipse region. The fitted column densities too are similar between the two observations with 
Table 3. The X-ray flux and fitted column density in various X-ray observations of XY Ari.

\begin{tabular}{llccr}
\hline \hline Mission & Date & $\begin{array}{c}2-10 \mathrm{keV} \mathrm{flux} \\
/ 10^{-11} \mathrm{erg} \mathrm{cm}^{-2} \mathrm{~s}^{-1}\end{array}$ & $\begin{array}{c}N_{\mathrm{H}} \\
/ 10^{22} \mathrm{~cm}^{-2}\end{array}$ & Ref. \\
\hline Ginga & 1989 Aug. & 2.5 & $3-16$ & Kamata \& Koyama (1993) \\
ASCA & 1995 Aug. & 1.79 & $3.9-4.4$ & this work \\
ASCA & 1996 Jan. & 1.84 & $4.3-4.5$ & this work \\
$R X T E$ & 1996 Jul. & 1.5 & $\sim 6-12$ & Hellier et al. (1997) \\
$R X T E$ & 1996 Jul. (o/b) & 5.5 & $\sim 40$ & Hellier et al. (1997) \\
$C h a n d r a$ & 2000 Jul. & 1.8 & $4.6-5.6$ & Salinas \& Schlegel (2004) \\
$X M M$ & 2000 Aug. & 1.13 & $4.2-4.6$ & Nb. incomplete orbital coverage \\
$X M M$ & 2001 Feb. & 1.20 & $4.6-5.0$ & this work \\
$R X T E$ & 2005 Nov. & 1.45 & $4.1-6.4$ & this work \\
$R X T E$ & 2006 Jan. & 1.32 & $5.4-8.1$ & this work \\
\hline
\end{tabular}

values of $N_{\mathrm{H}}=4.4 \pm 0.2 \times 10^{22} \mathrm{~cm}^{-2}$ and $N_{\mathrm{H}}=4.8 \pm 0.2 \times$ $10^{22} \mathrm{~cm}^{-2}$ respectively.

\section{Discussion}

In the RXTE observations of XY Ari presented here, we see two unprecedented changes in its behaviour: the previously seen broad orbital modulation has essentially disappeared, and its double-peaked spin pulse profile is no longer dominated by phase varying photoelectric absorption. The archival observations from ASCA and XMM-Newton allow us to investigate these changes in the context of its long term behaviour. They show that the extent and phasing of the broad orbital modulation is variable on timescales of $\sim$ months to years and also that the pulse profile varies between double and single peaked, even when the system is not undergoing an outburst. The alteration to the orbital modulation implies that changes have occurred in the outer regions of the accretion disc, whilst the alteration to the spin pulse profile implies that changes have occurred to the accretion curtains close to the white dwarf surface. We now consider possible reasons for each change in behaviour and seek to find a common cause for them.

\subsection{A continued decline from outburst?}

In addition to the changing orbital modulation and spin pulse profile that we have reported here, another feature that sets XY Ari apart from many other IPs is the occurence of at least one X-ray outburst, observed in July 1996. Although the nature of outbursts in magnetic CVs is debated (e.g. Hellier et al. 1997, 2000), if they are similar to those of conventional dwarf novae (Cannizzo 1993), it is likely that their recurrence timescale are lengthened as a result of the inner disc being truncated by the white dwarf's magnetic field (e.g. Matthews et al. 2007). The $\sim 30$ yr recurrence period of the outbursts in WZ Sge is explained satisfactorily by a magnetic accretor model (Matthews et al. 2007), so a >10 yr recurrence period in XY Ari is not unreasonable.

In order to assess the possibility of a link between a varying mass accretion rate and the modulation changes we see, we consider how the observed flux and measured column density to XY Ari have changed over the course of its various observations. These are summarised in Table 3, where it is clear that there is little evidence for a significant change in flux following the 1996 outburst. The average flux before the outburst is $\sim 1.9 \times 10^{-11} \mathrm{erg} \mathrm{cm}^{-2} \mathrm{~s}^{-1}$, and the average flux after the outburst is $\sim 1.4 \times 10^{-11} \mathrm{erg} \mathrm{cm}^{-2} \mathrm{~s}^{-1}$, which might reflect a $\sim 30 \%$ decrease in the mass accretion rate. However, as all these flux measurements are dependent on the spectral model used to fit the data in each case, and different instruments were used for each measurement, the variations are not necessarily significant. Similarly, the column density, apart from during the outburst itself, has remained fairly constant throughout these observations at about $4-5 \times 10^{22} \mathrm{~cm}^{-2}$.

Smith et al. (2005) show that the hydrogen column density due to the MBM12 molecular cloud varies across the cloud between $2-15 \times 10^{21} \mathrm{~cm}^{-2}$. This suggests that a column density of at least $3 \times 10^{22} \mathrm{~cm}^{-2}$ is intrinsic to XY Ari. However, the value we get from a simple bremsstrahlung fit to the $\mathrm{X}$-ray spectrum is not necessarily the correct one. It is likely that XY Ari, as in most IPs, has a spectrum which may be described by two complex, partial covering, absorbers: one for the accretion curtain and one for the dip-causing material at the outer edge of the accretion disc. Lower accretion rates will reduce both complex abosorbers, in terms of their column density and/or their covering fraction. Because the molecular cloud reduces our effective bandpass, it is not possible to do a complex absorber fit to these faint spectra, so we use a simple absorbed bremsstrahlung instead. Such fits can tell us in qualitative terms when the amount of absorber was higher or lower, but it is not possible to interpret the column density values obtained in a simple way.

If the change in flux is real then, given that the magnetospheric radius is a function of the accretion rate $\left(R_{\text {mag }} \propto \dot{M}^{-2 / 7}\right)$ this might imply an increase in the magnetospheric radius by about $10 \%$. That in turn might allow the lower magnetic pole of the white dwarf to be more visible, as well as reducing the contribution from the impact region at the outer edge of the disc. These two changes together could then conceivably explain some of the changes in the broad orbital modulation and some of the changes in the spin pulse profile that are seen. However, those modulations do not simply change systematically from before to after the outburst. We therefore conclude that there is no compelling evidence for a significantly altered X-ray flux or column density between any of the various X-ray observations of XY Ari in quiescence. So we must look for other causes of the changes in orbital modulation and spin pulse profile.

\subsection{A changing orbital modulation}

The broad orbital modulation seen from XY Ari was prominent when the object was observed by Ginga, ASCA, Chandra and XMM-Newton between 1989 and 2000. By the time of the second XMM-Newton observation in 2001, the broad modulation was less apparent with a significant decrease in the low energy modulation depth. In the most recent observations, with $R X T E$, the broad modulation is no longer detected with any significance. We note also that the phase of minimum of the broad 
modulation appears to drift significantly from one observation to another when the modulation itself is prominent.

Since the broad modulation is believed to be due to absorption by an azimuthally localised structure near the edge of an accretion disc, its disappearance suggests one of three possibilities: the absorbing structure may have diminished, extended to all azimuths, or otherwise moved out of our line of sight, at the time of these observations. As noted earlier, a similar effect has previously been seen in AO Psc, where an orbital modulation was seen by EXOSAT (1983 and 1985), Ginga (1990), ROSAT (1994) and ASCA (1994) but not by RXTE (1997); whereas for V1223 Sgr an orbital modulation was seen by EXOSAT (1983 and 1984), not seen by Ginga (1991) or ROSAT (1994), then seen again by ASCA (1994) and RXTE (1998) (Parker et al. 2005). In that earlier paper, we preferred the third of the possibilities above as the likely cause and suggested that the varying appearence of such orbital modulations may be due to the precession of a tilted accretion disc. This might explain both the drift of the phase of minimum and the ultimate disappearence of the modulation on long timescales as the extended bulge at the outer edge of the accretion disc moves out of our line of sight to the X-ray source.

One IP, TV Col, is known to have a $4 \mathrm{~d}$ photometric variation which is assumed to represent a disc precession period (Barrett et al. 1988), and similar effects are seen in several X-ray binaries. The Low Mass X-ray Binary system 4U1916-053 displays X-ray "dips" as its tilted disc periodically obscures the central X-ray source (Homer et al. 2001) and "superorbital" X-ray periods have been noted in systems such as Her X-1, SS433 and LMC X-4, which are also explained by precessing tilted or warped accretion discs (e.g. Clarkson et al. 2003).

Smoothed Particle Hydrodynamic simulations of accretion discs irradiated by a central X-ray source have been shown to induce twists or warps into the discs, which tilt and then precess with periods of a few days (Foulkes et al. 2006). These authors ran simulations for systems with a range of mass ratios and X-ray luminosities and showed that radiation-driven warping occurred in all cases. For systems with a relatively high mass ratio, such as XY Ari with $0.43<q<0.71$, the entire disc tilts out of the orbital plane, and then precesses. Foulkes et al. (2006) showed that the rate of precession is faster, and the tilt is larger, for higher X-ray luminosities. Whilst the mass ratio of XY Ari fulfils the necessary criterion, its X-ray luminosity $\left(L_{\mathrm{X}} / L_{\text {Edd }} \sim 5 \times 10^{-6}\right.$ ) is significantly lower than any system they modelled and is unlikely to be strong enough to drive the warp (Foulkes, private communication).

However, Foulkes et al. did not take into account any interaction between the compact object's magnetic field and the accretion disc. It is conceivable that, in a system such as XY Ari where the white dwarf's magnetic field is inclined with respect to the disc plane, the field lines attaching to the inner (truncated) disc edge may themselves serve to induce a warp in the disc as they rotate with respect to the disc material. In this case, such warps may be common amongst IPs and we encourage the construction of simulations to investigate this possibility.

The fact that we see no evidence for changes in the X-ray orbital modulation of XY Ari on the $\sim 1$ month timescale which corresponds to the gap between the two halves of the RXTE observation suggests that the disc precession period in XY Ari may be rather long - perhaps of order months. Although the presence of the broad modulation would be expected to come and go on the timescale of the precession period, we note that the broad modulation itself would actually occur on the beat period between the precession and orbital periods. Such a modulation is indeed seen in TV Col at a period of $5.2 \mathrm{~h}$, which is the beat between the $5.5 \mathrm{~h}$ orbital period and the $4 \mathrm{~d}$ retrograde disc precession period (Hellier 1993). However, with a precession period of (say) $>100 \mathrm{~d}$, the beat period in XY Ari would only differ from the orbital period by $<0.25 \%$ and so would be essentially indistinguishable from it in this case. Hence we cannot use the broad modulation (when present) to measure the precession period.

\subsection{A changing spin pulse profile}

If the changing orbital modulation in XY Ari really is due to a precessing disc, the next question to address is whether this can explain the observed variations in the spin pulse profile too.

On its discovery by Ginga in 1989, XY Ari exhibited a double-peaked pulse profile which showed a decreasing modulation depth with increasing energy, up to $10 \mathrm{keV}$ (Kamata \& Koyama 1993). Such a pulse profile, which is seen in several IPs, has been interpreted as indicating emission from two accreting poles, both of which are visible (Norton et al. 1999; Hellier et al. 1997). The observed variation in modulation depth with energy is as expected if the modulation is produced by phase-varying photoelectric absorption close to the surface of the white dwarf in the accretion curtains. However, we note that the lowest energy pulse profile presented by Kamata \& Koyama (1993) (i.e. below $\sim 5 \mathrm{keV}$ ) shows a structure in which the "second" peak is significantly dimished, indicating that the absorbing structure is different for the two accretion poles in this case. When a similar double-peaked pulse profile was seen by RXTE observations of XY Ari both before and after its 1996 outburst, Hellier et al. (1997) explained the low amplitude of the pulse by attributing it to an asymmetry between the emission at the two poles, without which cancellation would occur and a constant flux would result. The only other previously published observations of XY Ari in quiescence are those obtained by Chandra in 2000. Salinas \& Schlegel (2004) show that the pulse profile in these observations is rather noisy, and although there is evidence for a varying hardness ratio with phase, the profile shape is essentially singlepeaked.

During the 1996 outburst observed by RXTE, the modulation depth of XY Ari's spin pulse was greatly enhanced and the profile changed from its former double-peaked structure to a smoothly sinusoidal profile (Hellier et al. 1997). The interpretation placed on this was that the inner disc extended closer to the white dwarf during outburst, so hiding the lower magnetic pole. After the outburst, the truncation radius of the disc increased once more, revealing the lower pole and so allowing the "usual" double-peaked pulse profile, dominated by absorption effects at both poles, to return.

In contrast to these previous observations, the spin pulse profiles seen in our RXTE observations of XY Ari from 2005/2006 are clearly double-peaked, but show no evidence for energy dependence, and therefore indicate that the dominant cause of the spin modulation at this time is not photoelectric absorption, but must be essentially geometric.

We further note the different pulse profiles seen during the ASCA and XMM-Newton observations which we report here for the first time. Although the ASCA observations preceded Hellier et al.'s (1996) RXTE observations of XY Ari in quiescence by only a few months, the pulse profile was either single-peaked or absent in those observations. Furthermore, although the XMMNewton observations followed that with Chandra by only a few months, the pulse profile observed there is clearly double-peaked above $2 \mathrm{keV}$. These changes are summarised in Table 1.

It is apparent from the above discussion that the visibility of the two accretion poles in XY Ari can change on a timescale 
of months or less. One way of accomplishing this may be for the white dwarf itself to precess, such that the magnetic field axis presents a varying inclination angle to the orbital plane. Tovmassian et al. (2007) have recently presented evidence for such behaviour in the probable IPs FS Aur and HS 2331+3905. The rapidly spinning white dwarfs in these systems (with spin periods of $\sim 100 \mathrm{~s}$ and $67.2 \mathrm{~s}$ respectively) have precession periods of just a few hours. Schwarzenberg-Czerny (1992) estimated that the white dwarf precession period would be $\sim 10^{4}-10^{5} \times$ the white dwarf spin period in typical IPs, implying a precession period of $\sim 24-240 \mathrm{~d}$ in XY Ari, although the numerical calculations of Leins et al. (1992) presented graphically by Tovmassian et al. (2007) suggest a shorter precession period of just a few days in this case. However, we note that we see no change in the X-ray lightcurves between the two RXTE visits reported here (separated by $\sim 50 \mathrm{~d}$ ) nor do we see any change on a timescale of $\sim 4 \mathrm{~d}$ by subdividing the visits (although the signal-to-noise ratio is then greatly reduced).

However, rather than postulate a precessing white dwarf and a precessing accretion disc, it is possible that the disc itself could hide the lower accreting pole to varying degrees at different times, if it is indeed tilted. Occasional hiding of the lower pole by the inner edge of the disc could certainly explain the appearance of a single peaked pulse profile (as seen in the ASCA and Chandra observations), using the same model as suggested by Hellier et al. (1997) to explain the single-peaked pulse profile seen in outburst. As the top of the accretion curtains will be anchored at the inner edge of the disc, we suggest that a precessing disc could also cause the absorbing material in the curtains to be largely removed from our line of sight to the emission regions, so giving rise to the energy-independent pulse profiles we see in the latest $R X T E$ observations.

\section{Conclusion}

We have demonstrated that the RXTE observations of XY Ari from November 2005 and January 2006 reveal its broad X-ray orbital modulation to have disappeared. At the same time, its spin pulse profile displays no evidence of phase-varying photoelectric absorption. Both of these features are in contrast to earlier observations of the source dating back to fifteen years earlier. However, observations with other X-ray satellites over the intervening years have revealed that the broad orbital modulation drifts in both phase and modulation depth, and that the spin pulse profile varies too in both shape and significance. However, there is no evidence for a systematic change in X-ray flux or column density of XY Ari (as the disc presumably settles into quiescence following its 1996 outburst) to explain the observed changes in behaviour. Instead we suggest that a precessing, tilted accretion disc may cause both the changes in the broad orbital modulation and the changes in the pulse profile. At its outer edge, the precessing disc will move the absorbing bulge in orbital phase and may remove it from our line of sight entirely. At its inner edge, the precessing disc will alter our view through the accretion curtains that are anchored there, and may also hide our view of the lower pole. The tilt and precession of the disc may be induced by the action of the inclined, rotating magnetic field lines at the disc's inner edge; it is unlikely to be driven by radiation from the central source.

We conclude by noting that since XY Ari is the only deeply eclipsing IP, in this source we are probing matter which is less than $6^{\circ}$ above the orbital plane. Other IPs may also possess precessing, tilted accretion discs. However, the influence of such a structure is more apparent in XY Ari than in other systems where we see an X-ray orbital modulation, because in those cases our line of sight is up to $30^{\circ}$ above the orbital plane. With the additional characteristic of showing outbursts on a timescale which may be $\sim 10 \mathrm{yr}, \mathrm{XY}$ Ari therefore allows us a unique view of the accretion disc structure amongst magnetic cataclysmic variables.

Acknowledgements. The research described here has made use of data from NASA's High Energy Astrophysics Science Archive Research Center. Astrophysics Research at the Open University is supported by PPARC Rolling Grant PP/D000963/1. We thank Dr. S. Foulkes for useful discussions and the anonymous referee for several interesting suggestions.

\section{References}

Allan, A., Hellier, C., \& Ramseyer, T. F. 1996, MNRAS, 282, 699 Barrett, P., O'Donoghue, D., \& Warner, B. 1988, MNRAS, 233, 759 Beardmore, A. P., Mukai, K., Norton, A. J., Osborne, J. P., \& Hellier, C. 1998, MNRAS, 297, 337

Cannizzo, J. 1993, ApJ, 419, 318

Clarkson, W. I., Charles, P.A., Coe, M. J., \& Laycock, S. 2003, MNRAS, 343, 1213

Ezuka, H., \& Ishida, M. 1999, ApJS, 120, 277

Foulkes, S. B., Haswell, C. A., \& Murray, J.R. 2006, MNRAS, 366, 1399

Halpern, J. P., \& Patterson, J. 1987, ApJ, 312, L31

Hellier, C. 1993, MNRAS, 264, 132

Hellier, C. 1997, MNRAS, 291, 71

Hellier, C., Mukai, K., \& Beardmore, A. P. 1997, MNRAS, 292, 397

Hellier, C., Kemp, J., Naylor, T., et al. 2000, MNRAS, 313, 703

Hellier, C., Harmer, S., \& Beardmore, A. P. 2004, MNRAS, 349, 710

Homer, L., Charles, P.A., Hakala, P., et al. 2001, MNRAS, 322, 827

Kamata, Y., \& Koyama, K. 1993, ApJ, 405, 307

Kamata, Y., Tawara, Y., \& Koyama, K. 1991, ApJ, 379, L65

Koyama, K., Takano, S., Tawara, Y. et al. 1991, ApJ, 377, 240

Leins, M., Soffel, M. H., Lay, W., \& Ruder, H. 1992, A\&A, 261, 658

Littlefair, S., Dhillon, V., \& Marsh, T. R. 2001, MNRAS, 327, 669

Matthews, O. M., Speith, R., Wynn, G. A., \& West, R.G. 2007, MNRAS, 375, 105

Norton, A. J., Beardmore, A. P., Allan, A., \& Hellier, C. 1999, A\&A, 347, 203

Norton, A. J., Wynn, G. A., \& Somerscales, R.V. 2004, ApJ, 614, 349

Parker, T.L., Norton, A.J., \& Mukai, K. 2005, A\&A, 439, 213

Patterson, J. 1994, PASP, 106, 209

Patterson, J., \& Halpern, J. P. 1990, ApJ, 361, 173

Salinas, A., \& Schlegel, E. M. 2004, AJ, 128, 1331

Schwarzenberg-Czerny, A. 1992, A\&A, 260, 268

Smith, R. K., Edgar, R. J., Plucinsky, P.P., et al. 2005, ApJ, 623, 225

Takano, S., Koyama, K., Tawara, Y., et al. 1989, IAUC 4745

Terada, Y., Ishida, M., \& Makishima, K. 2004, PASJ, 56, 533

Tovmassian, G. H., Zharikov, S. V., \& Neustroev, V.V. 2007, ApJ, 655, 466

Warner, B. 1995, Cataclysmic Variable Stars, Cambridge Astrophysics Series (Cambridge, New York: Cambridge University Press)

Watson, M. G., King, A. R., \& Osborne, J. P. 1985, MNRAS, 212, 917 